\title{
Modelo de regressão linear múltipla aplicado a dados para geração de energia
}

\section{através do biogás}

\author{
Multiple linear regression model applied to data for biogas power generation \\ Modelo de regresión lineal múltiple aplicado a datos para la generación de energía a través del
}

biogás

\author{
Matheus da Silva Carvalho \\ ORCID: https://orcid.org/0000-0001-8758-3058 \\ Centro Universitário de Volta Redonda, Brasil \\ E-mail: matheuscarvalhos722@gmail.com
}

\begin{abstract}
Resumo
A industrialização mundial vem crescendo em larga escala, com isso fazendo com que a demanda de energia global também aumente. Acredita-se que para haver maior disponibilidade de energia, será necessário exigir muito mais do meio ambiente. Como algumas técnicas utilizadas para obtenção de energia são extremamente agressivas ao ecossistema, as fontes de energia renováveis desempenham um papel importantíssimo, comparado aos efeitos de obtenção de energia e sua relação a outras fontes de energia. A biomassa tem recebido muita atenção por parte da mídia e de líderes políticos, tanto em relação a fontes alternativas de combustível quanto uma maneira de minimizar a dependência da importação de matéria-prima de países desenvolvidos. Este trabalho utiliza um método de modelagem usando comparativos do software CH4 Biogás Simulator para avaliação de múltiplas variáveis afim de determinar a equação de regressão, assim obtendo a quantidade de biogás bruto e energia elétrica que se pode gerar utilizando dejetos de bovinos, suínos e aves pela ação de biodigestores. A equação gerada é de fácil aplicabilidade e resultou no modelo $\mathrm{Y}=1,572731+0,985985$ x bovinos $+0,191372$ x suínos $+0,021287$ x aves com $(\mathrm{r}=0,99)$ sendo assim adequada para estimação do valor do potencial de biogás.
\end{abstract}

Palavras-chave: Energia; Biomassa; Biogás; Biodigestores.

\begin{abstract}
World industrialization has been growing on a large scale, thereby causing the global energy demand to increase as well. That said, in order to have more energy available, much more will be required from the environment. Because some energy-using techniques are extremely aggressive to the ecosystem, renewable energy sources play a major role compared to the effects of energy generation and their relationship to other energy sources. Biomass has received much attention from the media and political leaders, both regarding alternative fuel sources and a way to minimize dependence on raw material imports from developed countries. This research uses a modelling method using $\mathrm{CH} 4 \mathrm{Biogas} \mathrm{Simulator}$ comparatives for multi-variable evaluation in order to determine the regression equation, thus obtaining the amount of crude biogas and electrical energy that can be generated using biogas from cattle manure, pigs and poultry by means of biodigesters. The generated equation is easy to apply and evolve in model $\mathrm{Y}=1.572731+0.985985 \mathrm{x}$ cattle +0.191372 $\mathrm{x}$ pigs $+0.021287 \mathrm{x}$ birds with $(\mathrm{r}=0.99)$ and is therefore suitable for estimating the potential value of biogas.
\end{abstract}

Keywords: Energy; Biomass; Biogas; Biodigesters.

\section{Resumen}

La industrialización mundial ha ido creciendo a gran escala, lo que ha provocado que también aumente la demanda mundial de energía. Se cree que para tener una mayor disponibilidad de energía será necesario exigir mucho más al medio ambiente. Como algunas técnicas utilizadas para obtener energía son extremadamente agresivas para el ecosistema, las fuentes de energía renovables juegan un papel muy importante, en comparación con los efectos de la obtención de energía y su relación con otras fuentes de energía. La biomasa ha recibido mucha atención de los medios de comunicación y los líderes políticos, tanto en términos de fuentes de combustibles alternativos como en una forma de minimizar la dependencia de materias primas importadas de países desarrollados. Este trabajo utiliza un método de modelado utilizando comparativos del software CH4 Biogas Simulator para evaluar múltiples variables con el fin de determinar la ecuación de regresión, obteniendo así la cantidad de biogás crudo y energía eléctrica que se puede generar utilizando estiércol bovino, porcino y avícola por acción de biodigestores. La ecuación generada es fácil de aplicar y resultó en el modelo $\mathrm{Y}=1.572731+0.985985 \mathrm{x}$ bovinos $+0.191372 \mathrm{x}$ porcinos $+0.021287 \mathrm{x}$ aves de corral con $(\mathrm{r}=$ $0.99)$ siendo por tanto adecuado para estimar el valor del potencial de biogás.

Palabras clave: La energia; Biomasa; Biogás; Biodigestores. 


\section{Introdução}

Com o passar dos anos, houve grande aumento da preocupação em relação ao meio ambiente, graças as consequências visíveis do desequilíbrio e degradação ambiental provocados pela interferência humana na natureza. Um dos principais responsáveis pelo desmatamento está na busca insaciável de recursos por parte das empresas, sem averiguar normas ambientais apropriadas.

Desta forma, vendo que a energia renovável é algo que advém dos ciclos naturais existentes em nosso planeta, a sua implementação, ajuda na substituição das unidades geradoras que não tem recursos renováveis. A energia renovável possui a capacidade de regeneração por meios naturais e contém uma vasta quantidade de energia, por essa razão, são conhecidas como fontes naturais virtualmente inesgotáveis (Medina, et al., 2016, p. 15). Dentre estas formas alternativas de energia, podemos citar a eólica, hidroelétrica, biomassa, geotérmica, das ondas e marés e os biocombustíveis.

O Brasil possui grande potência na produção de biocombustíveis em virtude do vasto tamanho territorial, por exemplo, gerando grandes porções de sobras de biomassa processados, no país essa quantidade é enorme e isso acaba ajudando muito compondo um grau importante na matriz elétrica. Iniciativas diplomáticas entre países visam expandir a participação de biocombustíveis na matriz energética global, desta forma, o Brasil possui melhores chances de se tornar um modelo de aplicação de fontes alternativas de energia (O GLOBO, 2018). Vasconcelos (2002, p. 72) analisa a biomassa sendo um recurso renovável de origem comum e orgânica sendo possível de ser transformada em energia mecânica, térmica ou elétrica. As principais substâncias orgânicas (matérias-primas) utilizadas na produção de biogás são: esterco proveniente das fezes animais (de bois, porcos, aves); bagaço da cana-de-açúcar; resíduos florestais (VG Resíduos, 2017).

A maior ênfase das fontes renováveis seria a biomassa, sendo que essa parcela correspondente mudou com o tempo, sendo composta no seu período inicial somente por carvão vegetal e lenha, e atualmente já fazem parte etanol de cana, biodiesel e dejetos de animais (CEMIG, 2012, p.296).

As energias convencionais (não renováveis) geram a perda de biodiversidade, chuva ácida, derramamento de óleo, poluição urbana (Goldemberg, 2007, p.12). A energia renovável é uma escolha super inteligente para os seres humanos e o meio ambiente. Aqui estão alguns dos grandes benefícios do uso de fontes renováveis para o nosso suprimento de energia (Solar Schools, 2019).

- Reduzem o efeito estufa pelo fato de não produzirem gases poluentes e $\mathrm{CO} 2$ na atmosfera;

- As energias renováveis não geram resíduos difíceis de tratar, como no caso da energia nuclear;

- $\quad$ As energias renováveis são inesgotáveis;

- $\quad$ Pode ser fabricada em casa evitando a dependência externa;

- As energias renováveis criam cinco vezes mais empregos que as convencionais.

Apesar de a energia renovável possuir muitos benefícios, deve-se citar algumas desvantagens em relação as fontes de combustíveis tradicionais, como se refere a seguir (Vitanna, 2019).

- $\quad$ Elas produzem altos impactos visuais;

- Você nem sempre obtém a mesma energia com eles, isto é, eles dependem do vento ou da quantidade de sol. Então, às vezes, têm dificuldades para garantir o suprimento e precisam ser complementados com outros exemplares de energia;

- Grandes extensões de terra são necessárias para obter uma quantidade apreciável de energia. 
O biogás é o metano formado por digestão anaeróbica. Capturá-lo e usá-lo tem o duplo benefício de reduzir as emissões diretas de GEE e fornecer uma fonte de energia renovável (Schiffer, 2016, p. 220). A Companhia Ambiental do estado de São Paulo (2019) afirma que o biogás é uma mistura de diversos gases, estabelecido em sua maior proporção por hidrocarbonetos como o metano ( $\mathrm{CH} 4)$ e dióxido de carbono ( $\mathrm{CO} 2)$, mas também possui em menores proporções outros gases como hidrogênio (H2), nitrogênio (N2), amônia (NH3), gás sulfídrico (H2S), oxigênio (O2). Sendo que dentre todos esse componentes citados em medidas estatísticas ele é composto por metano (50\% a 75\%) e dióxido de carbono (Aneel, 2002).

O biogás possibilita a geração de energia elétrica (reduzindo os gastos com energia) ou pode ser adaptado para itens domésticos a gás como o fogão, e não emite poluentes gasosos (Lumi, 2014). A biodigestão deixa resíduos sólidos e líquidos cheios de nutrientes (N, P, K, Ca, etc.) que poderia ser usado na melhora da qualidade do solo e no cultivo de plantas (Tunes, 2017, p. 13).

Devido a dificuldade de acesso por parte da população e a falta de certos recursos a uma forma de analisar facilmente e satisfatoriamente a quantidade de energia elétrica que se pode obter através de dejetos de animais, este trabalho foi realizado, possibilitando uma análise satisfatória e de fácil utilização através da fórmula de regressão linear múltipla.

\section{Metodologia}

As informações do trabalho dar-se através de pesquisas relacionadas as variáveis que envolvem a quantidade de energia que pode ser gerada através da digestão anaeróbica de resíduos de bovinos, suínos e aves da mesma em um biodigestor, com aplicação de modelo em uma fazenda para consumo próprio, obtendo também uma melhoria ecológica e econômica do sistema.

Pode-se afirmar, ainda, que esse estudo também é de caráter bibliográfico, constituindo-se de um estudo de casos que se da por meio da investigação e interpretação de livros, artigos e teses, que constam nas Referências deste artigo e do método de Regressão linear múltipla, que possui o objetivo de obter uma equação que explique satisfatoriamente a relação entre uma variável resposta Y (dependente) e uma e/ou mais variáveis explicativas X (independentes), possibilitando fazer a predição de valores de interesse (Guimarães, 2012).

Caso considerarmos apenas uma variável dependente dizemos que é uma regressão linear simples, se for duas ou mais variáveis se torna uma regressão múltipla (Rodrigues, 2012).

De acordo com Guimarões (2012) estes modelos de regressão são amplamente utilizados em diversas áreas tais como: Engenharias, biologia, agronomia, tecnologia, saúde, administração, etc.

Para elaboração do projeto, foi necessário:

- Analisar as diferentes tecnologias utilizadas para tratamento do biogás;

- Utilização de uma planilha de cálculo de animais por hectare utilizando valores aleatórios de cada espécie pecuária para início da coleta de dados e modelagem da fórmula;

- Levantamento de dados de uma amostra de 11 tamanhos diferentes de fazendas onde foram avaliadas as seguintes variáveis: Estimativa do teor de metano disponível para geração de energia elétrica e aquecimento, quantidade de dejetos, potencial do biogás bruto, matéria orgânica e energia elétrica;

- Determinação da quantidade de biogás que pode ser produzido de acordo com a quantidade de porcos, gados e/ou aves, para deter a energia que poderá ser aproveitada. 


\section{Regressão Linear Múltipla}

A regressão linear múltipla é uma técnica utilizada como aproximação de funções. Não se sabe a verdadeira relação entre $\mathrm{Y}, \mathrm{x} 1$ e x2, porém, em certas faixas das variações independentes, o modelo linear é uma aproximação adequada (Larson, et al., 2012 \& Montgomery, et al., 2012).

- $\quad$ Expressão matemática

$Y=\beta_{0}+\beta_{1} x_{1}+\beta_{2} x_{2}+\ldots+\beta_{k} x_{k}+\varepsilon$

- $\quad$ Critério dos mínimos quadrados

$y_{i}=\beta_{0}+\beta_{1} x_{i 1}+\beta_{2} x_{i 2}+\ldots+\beta_{k} x_{i k}+\varepsilon_{i}$

- $\quad$ O sistema em forma de matriz

$\left|\begin{array}{ccccc}n & \sum_{i=1}^{n} x_{i 1} & \sum_{i=1}^{n} x_{i 2} & \ldots & \sum_{i=1}^{n} x_{i k} \\ \sum_{i=1}^{n} x_{i 1} & \sum_{i=1}^{n} x_{i 1}^{2} & \sum_{i=1}^{n} x_{i 1} x_{i 2} & \ldots & \sum_{i=1}^{n} x_{i 1} x_{i k} \\ \vdots & \vdots & \vdots & \ddots & \vdots \\ \sum_{i=1}^{n} x_{i k} & \sum_{i=1}^{n} x_{i k} x_{i 1} & \sum_{i=1}^{n} x_{i k} x_{i 2} & \ldots & \sum_{i=1}^{n} x_{i k}^{2}\end{array}\right|\left|\begin{array}{c}\beta_{0}^{\wedge} \\ \beta_{1}^{\wedge} \\ \vdots \\ \beta_{k}^{\wedge}\end{array}\right|=\left|\begin{array}{c}\sum_{i=1}^{n} y_{i} \\ \sum_{i=1}^{n} x_{i 1} y_{i} \\ \vdots \\ \sum_{i=1}^{n} x_{i k} y_{i}\end{array}\right|$

\section{Resultados e Discussão}

\section{Classificação das variáveis}

A variável Y do trabalho, isto é, a variável resposta, foi escolhida como a quantidade de biogás bruto que pode ser obtido a partir das variáveis independentes (x1, x2 e x3) do projeto; que são respectivamente as seguintes: Quantidade de bovinos, suínos e aves.

O número exato de animais varia conforme o tamanho e as condições da fazenda, mas procurando manter a viabilidade do projeto, será necessário que a de produção de biogás se mantenha em níveis adequados, conforme pode ser visto na Tabela 1.

Tabela 1: Produção de biogás a partir de resíduos pecuários.

\begin{tabular}{cccc}
\hline Especie pecuária & Unidade referência & $\begin{array}{c}\text { Produção Específica de Biogás } \\
(\mathrm{m} 3 / \mathrm{kg} \mathrm{SV})\end{array}$ & Produção Diária (m3/animal/dia) \\
\hline \multirow{2}{*}{ Bovinos } & Gado de corte $\sim 500 \mathrm{~kg}$ & 0,28 & 0,292 \\
& & & 0,45 \\
Suínos & Porco $(50 \mathrm{~kg})$ & $0,46-0,77$ & 0,799 \\
Aves & Galinha $(2,5 \mathrm{~kg})$ & $0,010-0,017$ \\
\hline
\end{tabular}

Fonte: Condebella (2006). 
A escolha dessas três variáveis foi feita devido a participação que tem no setor agropecuário do país. De acordo com o censo agropecuário de 2017 divulgado pelo Instituto Brasileiro de Geografia e Estatística (IBGE), cerca de R \$ 263,9 bilhões do PIB do país é proveniente da pecuária, sendo 73,7\% representada pela pecuária de corte.

A criação de suínos e aves para o abate pode ser feita em número consideravelmente maior em comparação ao do gado bovino, gerando mais empregos para suportar o tempo longo de produção. Como exemplo, o estado de Minas Gerais demonstrou no censo agropecuário de 2017 do IBGE um número de cabeças de suínos superior a 4.700 .000 enquanto que o rebanho de galinhas equivalia a aproximadamente 124.413.000.

\section{Coleta de Dados}

A metodologia desse trabalho foi realizada utilizando-se o Software CH4Biogassimulator, que simula a produção de biogás em propriedades rurais por unidade de criação de animais, apresentando um relatório que informa os ganhos energéticos, ambientais e econômicos afim de recolher a máxima quantidade de dados possível para se realizar a regressão (CH4 AGROENERGIA, 2019).

Foi montado uma planilha reunindo todos os dados úteis e relevantes demonstrando o que o aplicativo forneceu, sendo eles: Estimativa do teor de metano (CH4) disponível para geração de energia elétrica e aquecimento, Quantidade de dejetos (Kg/dia), Potencial do Biogás Bruto (Nm3/dia), Matéria Orgânica (Kg/ciclo) e Energia elétrica (Kwh/dia).

Para o cálculo da quantidade de animais foi utilizado uma planilha que a Boi Saúde - Pecuária Inteligente fornece, levando em conta a quantidade de consumo de animal por dia/ano calculado a partir dos dados da média do peso da espécie pecuária considerada (bovinos, suínos e aves) conforme mostra a Tabela 2 a seguir:

Tabela 2: Exemplo de cálculo de quantidade de animais para animais de 700 quilos em 100 hectares.

Planilha para cálculo da quantidade de animais por hectare

\begin{tabular}{lc}
\hline Média de produção de pastagem por ano & $15000 \mathrm{~kg} / \mathrm{hectare}$ \\
Média de consumo por animal & $4 \%$ do pesso vivo do animal \\
Média do peso dos seus animais & 700 quilos \\
Quantidade de consumo por animal de & 28 quilos por dia \\
Quantidade de consumo por animal de & 10220 quilos por ano \\
O ideal é ter aproximadamente & 1,47 animais por hectare \\
\hline Eu tenho & 100 hectares \\
Portanto, considerando o pasto, devo ter & 147 animais na minha propriedade
\end{tabular}

Fonte: Boi Saúde - Pecuária Inteligente (Adaptado pelos Autores).

Utilizou-se onze diferentes tamanhos de fazendas hipotéticas afim de analisar a variação de animal por cada hectare selecionado. A quantidade de animais utilizada por cada hectare escolhida não seguiu um padrão ou uma constante em todos tamanhos, pelo mesmo motivo de provocar esta variação e aumentar a probabilidade de êxito na construção da fórmula de regressão linear das variáveis múltiplas. O Quadro 1 a seguir demonstra como ficou essa divisão. 
Quadro 1: Quantidade de animais por tamanho de fazenda.

\begin{tabular}{|c|c|c|c|}
\hline Tamanho da fazenda (hectares) & Bovinos & Suínos & Aves \\
\hline 25,00 & 9,00 & 308,00 & 1233,00 \\
\hline 27,50 & 11,00 & 247,00 & 822,00 \\
\hline 30,00 & 13,00 & 205,00 & 4110,00 \\
\hline 32,50 & 27,00 & 164,00 & 1849,00 \\
\hline 35,00 & 13,00 & 144,00 & 7397,00 \\
\hline 37,50 & 14,00 & 308,00 & 4932,00 \\
\hline 40,00 & 17,00 & 257,00 & 6164,00 \\
\hline 42,50 & 27,00 & 216,00 & 4932,00 \\
\hline 45,00 & 20,00 & 329,00 & 5753,00 \\
\hline 47,50 & 25,00 & 411,00 & 3699,00 \\
\hline 50,00 & 23,00 & 308,00 & 7397,00 \\
\hline
\end{tabular}

Fonte: Autores.

\section{Análise de Dados}

Após a coleta da quantidade de dados de cada espécie pecuária por tamanho de fazenda, foram utilizados estes valores e inseridos no Software CH4Biogassimulator para realizar a equação de regressão linear múltipla. Sendo assim, foi escolhido como prioridade os dados recolhidos do potencial de biogás bruto. Conforme pode ser visto no Quadro 2, os resultados não foram crescentes, e isto propositalmente, pelo mesmo motivo de se obter esta variação e aumentar a credibilidade dos coeficientes independentes na fórmula final $Y=b 0+b 1 . x 1+b 2 . x 2+b 3 . x 3$.

Quadro 2: Análise da quantidade de biogás em $\mathrm{Nm}^{3} /$ dia.

\begin{tabular}{|c|c|c|c|}
\hline Y & x1 & x2 & x3 \\
\hline Potencial do Biogás bruto $\left(\mathrm{Nm}^{3} /\right.$ dia $)$ & Bovinos & Suínos & Aves \\
\hline 95,77 & 9,00 & 308,00 & 1233,00 \\
\hline 76,52 & 11,00 & 247,00 & 822,00 \\
\hline 140,78 & 13,00 & 205,00 & 4110,00 \\
\hline 100,23 & 27,00 & 164,00 & 1849,00 \\
\hline 199,49 & 13,00 & 144,00 & 7397,00 \\
\hline 179,88 & 14,00 & 308,00 & 4932,00 \\
\hline 198,91 & 17,00 & 257,00 & 6164,00 \\
\hline 172,93 & 27,00 & 216,00 & 4932,00 \\
\hline 207,01 & 20,00 & 329,00 & 5753,00 \\
\hline 183,43 & 25,00 & 411,00 & 3699,00 \\
\hline 240,89 & 23,00 & 308,00 & 7397,00 \\
\hline \multicolumn{4}{|c|}{ SOMA } \\
\hline 1795,84 & 199,00 & 2897,00 & 48288,00 \\
\hline 163,26 & 18,09 & 263,36 & 4389,82 \\
\hline \multicolumn{4}{|c|}{ MÉDIA } \\
\hline
\end{tabular}

Fonte: CH4Biogassimulator.

Para início da montagem da regressão linear, dividiu-se cada variável representado no Quadro 3 e foi realizado os seguintes cálculos. 
Quadro 3: Cálculo dos coeficientes da matriz 4x4 de regressão.

\begin{tabular}{|c|c|c|c|c|c|c|c|c|}
\hline $\mathrm{X}_{1}{ }^{2}$ & $\mathrm{X}_{2}{ }^{2}$ & $\mathrm{X}_{3}{ }^{2}$ & $\mathrm{X}_{1} \mathrm{X}_{2}$ & $\mathrm{X}_{1} \mathrm{X}_{3}$ & $\mathrm{X}_{2} \mathrm{X}_{3}$ & $\mathrm{yx}_{1}$ & $\mathrm{yx}_{2}$ & $\mathrm{yx}_{3}$ \\
\hline 81,00 & 94864,00 & 1520289,00 & 2772 & 11097 & 379764 & 861,93 & 29497,16 & 118084,41 \\
\hline 121,00 & 61009,00 & 675684,00 & 2717 & 9042 & 203034 & 841,72 & 18900,44 & 62899,44 \\
\hline 169,00 & 42025,00 & 16892100,00 & 2665 & 53430 & 842550 & 1830,14 & 28859,9 & 578605,8 \\
\hline 729,00 & 26896,00 & 3418801,00 & 4428 & 49923 & 303236 & 2706,21 & 16437,72 & 185325,27 \\
\hline 169,00 & 20736,00 & 54715609,00 & 1872 & 96161 & 1065168 & 2593,37 & 28726,56 & 1475627,53 \\
\hline 196,00 & 94864,00 & 24324624,00 & 4312 & 69048 & 1519056 & 2518,32 & 55403,04 & 887168,16 \\
\hline 289,00 & 66049,00 & 37994896,00 & 4369 & 104788 & 1584148 & 3381,47 & 51119,87 & 1226081,24 \\
\hline 729,00 & 46656,00 & 24324624,00 & 5832 & 133164 & 1065312 & 4669,11 & 37352,88 & 852890,76 \\
\hline 400,00 & 108241,00 & 33097009,00 & 6580 & 115060 & 1892737 & 4140,2 & 68106,29 & 1190928,53 \\
\hline 625,00 & 168921,00 & 13682601,00 & 10275 & 92475 & 1520289 & 4585,75 & 75389,73 & 678507,57 \\
\hline 529,00 & 94864,00 & 54715609,00 & 7084 & 170131 & 2278276 & 5540,47 & 74194,12 & 1781863,33 \\
\hline \multicolumn{9}{|c|}{ SOMA } \\
\hline 4037,00 & 825125,00 & 265361846,00 & 52906,00 & 904319,00 & 12653570,00 & 33668,69 & 483987,71 & 9037982,04 \\
\hline \multicolumn{9}{|c|}{ MÉDIA } \\
\hline 367,00 & 75011,36 & 24123804,18 & 4809,64 & 82210,82 & 1150324,55 & 3060,79 & 43998,88 & 821634,73 \\
\hline
\end{tabular}

Fonte: Autores.

\section{Conclusão}

Utilizando este esquema de matriz $4 \mathrm{x} 4$ e a seguinte fórmula do coeficiente de correlação, foi estimado o valor dos coeficientes b0, b1, b2, b3 e R. O Anexo 1 e 2 mostram como o resultado final foi alcançado.

$$
\begin{gathered}
\left|\begin{array}{cccc}
n & \Sigma x_{1} & \Sigma x_{2} & \Sigma x_{3} \\
\Sigma x_{1} & \Sigma x_{1}{ }^{2} & \Sigma x_{1} x_{2} & \Sigma x_{1} x_{3} \\
\Sigma x_{2} & \Sigma x_{1} x_{2} & \Sigma x_{2}{ }^{2} & \Sigma x_{2} x_{3} \\
\Sigma x_{3} & \Sigma x_{1} x_{3} & \Sigma x_{2} x_{3} & \Sigma x_{3}{ }^{2}
\end{array}\right| \times\left|\begin{array}{c}
b_{0} \\
b_{1} \\
b_{2} \\
b_{3}
\end{array}\right|=\left|\begin{array}{c}
\Sigma y \\
\Sigma x_{1} y \\
\Sigma x_{2} y \\
\Sigma x_{3} y
\end{array}\right| \\
R=\sqrt{\frac{\Sigma(y-\bar{y})^{2}}{\left(\Sigma y_{i}-\bar{y}\right)^{2}}}
\end{gathered}
$$

Segue abaixo no Quadro 4, a matriz 4x4 que demonstra a continuação dos cálculos realizados anteriormente. Os resultados obtidos através do mesmo correspondem aos coeficientes b0, b1, b2 e b3; obtendo como resposta os seguintes valores: b0=1,5727312; b1=0,9859849; b2=0,1913721 e b3=0,0212874. Esses são fundamentais para serem aplicados na fórmula final, de forma que não variam e se tornam valores fixos; mediante a escolha das variáveis do usuário da fórmula que esse projeto oferece. 
Quadro 4: Cálculo de obtenção dos coeficientes b0,b1,b2 e b3.

\begin{tabular}{|c|c|c|c|c|c|}
\hline $\mathrm{n}$ & $\Sigma \times 1$ & $\Sigma \times 2$ & $\Sigma \times 3$ & $\mathrm{~b} 0$ & $\Sigma \mathrm{y}$ \\
\hline$\Sigma \times 1$ & $\Sigma \times 12$ & $\Sigma \times 1 \times 2$ & $\Sigma \times 1 \times 3$ & $\mathrm{~b} 1$ & $\Sigma \times 1 \mathrm{y}$ \\
\hline$\Sigma \times 2$ & $\Sigma \times 1 \times 2$ & $\Sigma \times 22$ & $\Sigma \times 2 \times 3$ & $\mathrm{~b} 2$ & $\Sigma \times 2 \mathrm{y}$ \\
\hline$\Sigma \times 3$ & $\Sigma \times 1 \times 3$ & $\Sigma \times 2 \times 3$ & $\Sigma \times 32$ & $\mathrm{~b} 3$ & $\Sigma \times 3 \mathrm{y}$ \\
\hline 11 & 199 & 2897 & 48288 & $\mathrm{~b} 0$ & 1795,84 \\
\hline 199 & 4037 & 52906 & 904319 & $\mathrm{~b} 1$ & 33668,69 \\
\hline 2897 & 52906 & 825125 & 12653570 & $\mathrm{~b} 2$ & 483987,71 \\
\hline 48288 & 904319 & 12653570 & 265361846 & $\mathrm{~b} 3$ & 9037982,04 \\
\hline 11 & 199 & 2897 & 48288 & $\mathrm{~b} 0$ & 1795,84 \\
\hline 0 & 436,9091 & 496,636364 & 30745,18182 & $\mathrm{~b} 1$ & 1180,3118 \\
\hline 0 & 496,6364 & 62160,5455 & $-63733,27273$ & $\mathrm{~b} 2$ & 11028,757 \\
\hline 0 & 30745,18 & $-63733,2727$ & 53386305,64 & $\mathrm{~b} 3$ & 1154571 \\
\hline 11 & 199 & 2897 & 48288 & $\mathrm{~b} 0$ & 1795,84 \\
\hline 0 & 436,9091 & 496,636364 & 30745,18182 & $\mathrm{~b} 1$ & 1180,3118 \\
\hline 0 & 0 & 61596,0169 & $-98681,44757$ & $\mathrm{~b} 2$ & 9687,092 \\
\hline 0 & 0 & $-98681,4476$ & 51222775 & $\mathrm{~b} 3$ & 1071512,7 \\
\hline 11 & 199 & 2897 & 48288 & $\mathrm{~b} 0$ & 1795,84 \\
\hline 0 & 436,9091 & 496,636364 & 30745,18182 & $\mathrm{~b} 1$ & 1180,3118 \\
\hline 0 & 0 & 61596,0169 & $-98681,44757$ & $\mathrm{~b} 2$ & 9687,092 \\
\hline 0 & 0 & 0 & 51064679,9 & $\mathrm{~b} 3$ & 1087032,2 \\
\hline & & & & & \\
\hline
\end{tabular}

Fonte: Autores

A seguir no Quadro 5, são evidenciados os cálculos do coeficiente de determinação $\mathrm{R}^{2}$, indicado para medir a explicação da reta de regressão.

Quadro 5: Cálculo do coeficiente de determinação $\mathrm{R}^{2}$

\begin{tabular}{|c|c|c|c|c|c|c|}
\hline $\mathbf{y}$ & $\mathbf{x 1}$ & $\mathbf{x 2}$ & $\mathbf{x 3}$ & $\mathbf{y}(\mathbf{a j u s ~ t a d o})$ & $\mathbf{( y - y m ) 2}$ & (yajustado - ym)2 \\
\hline 95,77 & 9 & 308 & 1233 & 95,636517 & 4554,6547 & 4572,689621 \\
\hline 76,52 & 11 & 247 & 822 & 77,185683 & 7523,5122 & 7408,474977 \\
\hline 140,78 & 13 & 205 & 4110 & 141,11286 & 505,26866 & 490,4152552 \\
\hline 100,23 & 27 & 164 & 1849 & 98,939674 & 3972,5517 & 4136,870409 \\
\hline 199,49 & 13 & 144 & 7397 & 199,41071 & 1312,7446 & 1307,005332 \\
\hline 179,88 & 14 & 308 & 4932 & 179,30838 & 276,28484 & 257,6088952 \\
\hline 198,91 & 17 & 257 & 6164 & 198,73238 & 1271,0521 & 1258,419049 \\
\hline 172,93 & 27 & 216 & 4932 & 174,51995 & 93,544067 & 126,827442 \\
\hline 207,01 & 20 & 329 & 5753 & 206,72003 & 1914,2216 & 1888,931904 \\
\hline 183,43 & 25 & 411 & 3699 & 183,61823 & 406,90225 & 414,531481 \\
\hline 240,89 & 23 & 308 & 7397 & 240,65558 & 6026,6992 & 5990,357918 \\
\hline
\end{tabular}

Fonte: Autores

Obtendo-se uma soma de 27857,43596 e 27852,13228 correspondes a (y-ym)² e (yajustado-ym) ${ }^{2}$ respectivamente, e utilizando-se a fórmula apresentada no começo dessa conclusão foi obtido um valor de $\mathrm{R}^{2}=0,910$ e uma correlação forte positiva satisfatória $(0,8 \leq \mathrm{Rxy}<1)$, evidenciando a veracidade da equação.

A equação gerada foi Potencial de Biogás Bruto $(\mathrm{Nm3/dia})=1,572731+0,985985$ x bovinos + 0,191372 x suínos + $\mathbf{0 , 0 2 1 2 8 7} \mathbf{x}$ aves apresentando um $(\mathrm{r}=0,99)$ sendo assim adequada para estimação do valor do potencial de biogás. 
Conforme mostra o Quadro 6, o valor resposta do biogás pode ser comparado com a quantidade de energia elétrica. Comparando-se a soma e a média das duas variáveis dependentes obtemos mais uma equação: Energia Elétrica $($ Kwh/dia) =

\section{1,167143 x Potencial do Biogás Bruto (Nm3/dia).}

Quadro 6: Comparação de Energia Elétrica com quantidade de biogás.

\begin{tabular}{|c|c|c|c|c|}
\hline Energia Elétrica Kwh/dia & Potencial do Biogás bruto $\left(\mathrm{Nm}^{3} / \mathrm{dia}\right)$ & Bovinos & Suínos & Aves \\
\hline 82,06 & 95,77 & 9,00 & 308,00 & 1233,00 \\
\hline 65,56 & 76,52 & 11,00 & 247,00 & 822,00 \\
\hline 120,62 & 140,78 & 13,00 & 205,00 & 4110,00 \\
\hline 85,88 & 100,23 & 27,00 & 164,00 & 1849,00 \\
\hline 170,93 & 199,49 & 13,00 & 144,00 & 7397,00 \\
\hline 154,12 & 179,88 & 14,00 & 308,00 & 4932,00 \\
\hline 170,43 & 198,91 & 17,00 & 257,00 & 6164,00 \\
\hline 148,17 & 172,93 & 27,00 & 216,00 & 4932,00 \\
\hline 177,36 & 207,01 & 20,00 & 329,00 & 5753,00 \\
\hline 157,16 & 183,43 & 25,00 & 411,00 & 3699,00 \\
\hline 206,39 & 240,89 & 23,00 & 308,00 & 7397,00 \\
\hline \multicolumn{5}{|c|}{ SOMA } \\
\hline 1538,68 & 1795,84 & 199,00 & 2897,00 & 48288,00 \\
\hline \multicolumn{7}{|c|}{ MÉDIA } & 18,09 & 263,36 & 4389,82 \\
\hline
\end{tabular}

Fonte: $\mathrm{CH}_{4}$ Biogassimulator (Adaptado pelos autores).

\section{Fica como sugestão para trabalhos futuros:}

A avaliação de outras unidades de referências de espécies pecuárias e comparação com a mesma para ver qual pode fornecer mais benefícios levando em conta a produção diária de dejetos m³/animal/dia;

A utilização desta fórmula em uma planta de uma fazenda real que possui animais em uma quantidade satisfatória, analisando toda a carga que a mesma contém e a quantidade de energia que consome em média durante um mês, investigando a viabilidade da implementação da forma de energia por biogás na mesma.

\section{Agradecimentos}

A UniFOA, seu corpo docente e administração que proporcionaram a realização desta pesquisa, ao professor e orientador do trabalho de conclusão de curso Vagner Silva Guilherme pelo apoio.

\section{Referências}

Alves, M. O., et al. (2014). Biodigestores - Fonte Renovável de Energia. UniCesumar. 5 p. ISBN: 9788580847246

ANEEL (2002). Atlas de Energia Elétrica do Brasil. (3ª.ed.): ANEEL, 236 p. ISBN: 978-85-87491-10-7.

CETESB (2019). Biogás. https://cetesb.sp.gov.br/biogas/.

Ch4Agroenergia (2019). https://inovacao2020.wixsite.com/ch4agroenegia

Eleven Advantages and Disadvantages of Renewable Energy (2019). https://vittana.org/11-advantages-and-disadvantages-of-renewable-energy.

Goldemberg, J., \& Lucon, O. (2006-2007). Energias Renováveis: Um futuro sustentável: Revista Usp, 15 p.

Guimarães, P. R. B. (2012) Métodos Quantitativos Estatísticos. (1 ${ }^{\text {a }}$ ed.): IESDE BRASIL S.A., 256 p. ISBN: 978-85-387-3028-6.

Hydroeletrectricity - BP Statistical Review of World Energy (2019). https://www.bp.com/content/dam/bp/business-sites/en/global/corporate/pdfs/energyeconomics/statistical-review/bp-stats-review-2019-hydroelectricity.pdf. 
Research, Society and Development, v. 10, n. 16, e224101623968, 2021

(CC BY 4.0) | ISSN 2525-3409 | DOI: http://dx.doi.org/10.33448/rsd-v10i16.23968

Larson, R., \& Farber, B (2008). Estatística Aplicada. Pearson Education (2ª Edição).

Lumi, M., et al. (2014). Manual Básico de Biogás. (1ª ed.): Editora Univates, 70p. ISBN: 978-85-8167-073-7.

Medina, J. M., et al. (2016). Eficiencia Energética y producción de Energía Eléctrica a partir de Biogás. 148 p. Dissertação (Graduação). Departamento de Ingeniería Eléctrica - Instituto Especializado de Estudios Superiores Loyola.

Minas Gerais, Companhia Energética (2012). Alternativas Energéticas: Uma visão Cemig. Belo Horizonte: 373p. ISBN: 978-85-87-929-51-8

Montgomery, D. C., \& Runger, G. C (2012). - Estatística Aplicada e Probabilidade para Engenheiros. Editora LTC - (2 Edição).

O globo (2018). https://oglobo.globo.com/economia/brasil-tem-matriz-energetica-menos-poluente-do-mundo-segundo-agencia-internacional-23244753.

Rodrigues, S. C. A. (2012). Modelo de regressão linear e suas aplicações. 94 p. Relatório de Estágio (Mestrado). Universidade da Beira Interior.

Schiffer, H. W (2016). World Energy Resources. London: World Energy Council, 1028 p. ISBN: 9780946121588

Software CH4 - Biogas Simulator. Versão 2.3.3 [S. I.], 12 nov. (2015). https://apkpure.com/ch4-biogas-simulator/br.com.biogasch4.CH4BiogasSimulator.

Solarschools (2019). Advantages and Disadvantages of Renewable Energy. https://www.solarschools.net/knowledge-bank/renewable-energy/advantagesdisadvantages.

Tunes, C. R. (2017). Produção De Biogás A Partir Da Digestão Anaeróbica De Efluentes Orgânicos Em Reator Uasb. 2017. 75 p. Dissertação (Mestrado). Campus Universitário de Gurupi - Universidade Federal do Tocantins.

Vasconcelos, G. F. (2017) Biomassa: A energia do futuro. (1 ${ }^{\mathrm{a}}$ ed.): Senac, 166p. 